\title{
Epidemiology of Multiple Sclerosis in Iran: A Systematic Review
}

\author{
Masoud Etemadifar ${ }^{a, b}$ Sepideh Sajjadi ${ }^{b, c}$ Zahra Nasr $^{b, c}$

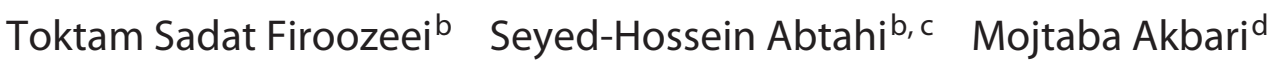 \\ Mahboobeh Fereidan-Esfahani ${ }^{b, c, e}$ \\ ${ }^{a}$ Department of Neurology, Medical School, ${ }^{b}$ Isfahan Research Committee of Multiple Sclerosis (IRCOMS), \\ 'Medical Students' Research Committee, 'Department of Epidemiology and Statistics, Isfahan University of Medical \\ Science, and e Persia Research Center, Sady Hospital, Isfahan, Iran
}

\section{Key Words}

Multiple sclerosis · Iran · Epidemiology · Incidence ·

Prevalence

\section{Abstract}

Background: There is a wide variation in the prevalence of multiple sclerosis (MS) in different geographical regions and the epidemiology of MS in Iran has been a major topic of concern during the last decade. Several population-based studies have shown a sharp increase in the prevalence and incidence of MS in this region. In this study, for the first time, the aim was to provide a comprehensive review regarding the incidence and prevalence of MS across Iran. Methods: A comprehensive literature search was performed using PubMed, Embase, and Web of Science. We also did a manual search of reference lists from primary articles and relevant reviews. Databases of ongoing research and unpublished literature were also searched. Results: A total of 22 relevant studies were reviewed and 11 studies met the inclusion criteria. Incidence data were found in 5 studies and ranged from 0.68 to $9.1 / 100,000$ per year in the Iranian population. Prevalence was reported in all studies and ranged from 5.3 to $74.28 / 100,000$ with the higher prevalence among females (female/male ratio ranged from 1.8 to 3.6). The most prevalent subtype of MS was the relapsing-remitting form (65.8$87.8 \%)$. The sensory disturbance was the most initial presentation. Conclusion: The incidence and prevalence of MS in Iran has been increasing rapidly, especially in females. Future research should focus on determining the epidemiological features of MS in the neglected provinces with different ethnicities. Such an effort along with further research towards improvement of data on previously studied areas can enable a field to be opened up to identify the patterns of MS in varied genetic backgrounds and environments of Iran.

(c) 2013 S. Karger AG, Basel

\section{Introduction}

Multiple sclerosis (MS) is a chronic inflammatory and immune-mediated demyelinating disease with complex etiology and still unknown causes, which affects individuals in their productive years [1]. There is a wide variation in the prevalence of MS in different geographical regions. About 30 years ago, Kurtzke [2,3] proposed that higher

\section{KARGER}

E-Mail karger@karger.com

www.karger.com/ene
(C) 2013 S. Karger AG, Basel

0014-3022/13/0706-0356\$38.00/0
Mahboobeh Fereidan-Esfahani

Isfahan MS Society

No. 2, Behesht Building, Bozorgmehr Ave.

Isfahan 81588-44799 (Iran)

E-Mail mf.esfahani@yahoo.com 
latitude increases the prevalence of MS and classified geographical areas for three regions by prevalence: (1) high $>30 / 100,000$, (2) intermediate (5-25/100,000), and (3) low risk $<5 / 100,000$. Based on such expectations, MiddleEastern countries had been generally classified as low-risk areas, but to date, several studies in this region report the higher risk [4].

Iran, a high desert plateau with high mountains, latitude $32^{\circ} 00^{\prime} \mathrm{N}$ longitude $53^{\circ} 00^{\prime}$, is a Muslim Middle-Eastern country with 31 provinces. Several ethnic groups, including Persians, Turks, Arabs, and Balochs, are now unified by Iranian culture. The majority speaks Persian, the official language.

For the last decade, the epidemiology of MS in this country has been a major topic of concern [5] and several population-based studies have shown a sharp increase in the prevalence and incidence of MS in this region. Iran's population has increased dramatically, rising from 17 million in 1950 to about 75 million by 2000, surpassing France, UK, and Italy.

At present, more than half of Iran's population is less than 35 years of age, born since 1977. The largest age group is $20-24$. MS mostly affects young adults $20-40$ years old, so the public health system is facing an increasing disease burden [6]. Any additional causes for such an increase are not clear, even as we consider the radical changes in lifestyle, now highly urbanized, and with vastly improved health care, with more MS cases diagnosed and reported [7]. To marshal data to sustain research, we present the first systematic review on the epidemiology of MS in Iran.

\section{Methods}

\section{Search Strategy}

We used computer databases to find population-based studies with data on MS epidemiology in Iran: PubMed, Embase, and Web of Science. We searched for papers published before August 1, 2013, using the following search terms: 'multiple sclerosis and Iran', 'multiple sclerosis and population', 'multiple sclerosis and epidemiology', 'multiple sclerosis and prevalence', 'multiple sclerosis and incidence'. All searches were repeated with 'demyelinating disease' in place of 'multiple sclerosis'. We also did a manual search of reference lists from primary articles and relevant reviews. If the search of international literature was unproductive, reliable evidence reported in non-English scientific literature or from local small population surveys was also used. No world language was explicitly excluded. Databases of ongoing research and unpublished literature were also searched. Our search strategy used a combination of controlled vocabulary with PubMed, and was adapted for the other databases.

\section{Inclusion and Exclusion Criteria}

The following criteria were used to select papers for inclusion in this systematic review: (1) the population was Iranian, defined primarily geographically as those living in Iran; (2) MS was defined according to accepted international diagnostic criteria in use at the time of the study, the Poser [8] or McDonald criteria [9], and (3) prevalence of MS was reported. Studies that were based in a single institution, or did not involve a network of hospitals serving a welldefined general population, were excluded.

\section{Data Extraction and Assessment of Study Quality}

Two of the authors agreed on the data to be extracted. Data included: study period, details of study population, and diagnostic selection criteria. One of the reviewers (Z.N.) extracted data using a data extraction form, while a second reviewer (M.F.-E.) confirmed the data. In cases of non-consensus, a third independent review (S.-H.A.) was obtained. In cases of weak study methodology, authors were approached to determine a study's potential inclusion. Each study was evaluated on the following items: (1) type of study design; (2) description of study population, and (3) adequacy of case definition, based on validated criteria. Those studies in which the items were not reported or unclear were excluded. The following data were extracted into table 1: (a) source: authors and journal published; (b) population denominator; (c) timescale: incidence time frame and prevalence date; (d) case ascertainment method; (e) diagnostic method, and (f) outcome: prevalence per 100,000 of population.

\section{Results}

\section{Existing Systematic Reviews}

No existing systematic reviews of incidence and prevalence were identified.

\section{Study Yield}

22 studies were identified. Of these, the following were excluded: 5 that reported the prevalence of MS among Iranian immigrants, 4 case reports, and 2 without prevalence and clinical patterns. Hence, 11 studies were included, published from 2006 to 2013 [10-20].

\section{Findings}

Table 1 illustrates a summary of results identified in the study.

\section{Tehran}

Tehran, the capital and most populated region of Iran, on the north of the central plateau, is expanding with mass immigration of people from all over the nation, with many different ethnic groups. Sahraian et al. [10] in 2010 used data from the Iran MS Registry 10-year national survey: prevalence 51.9, female/male ratio 2.60 , age at disease onset $27.2 \pm 8.3$. MS was more prevalent in 


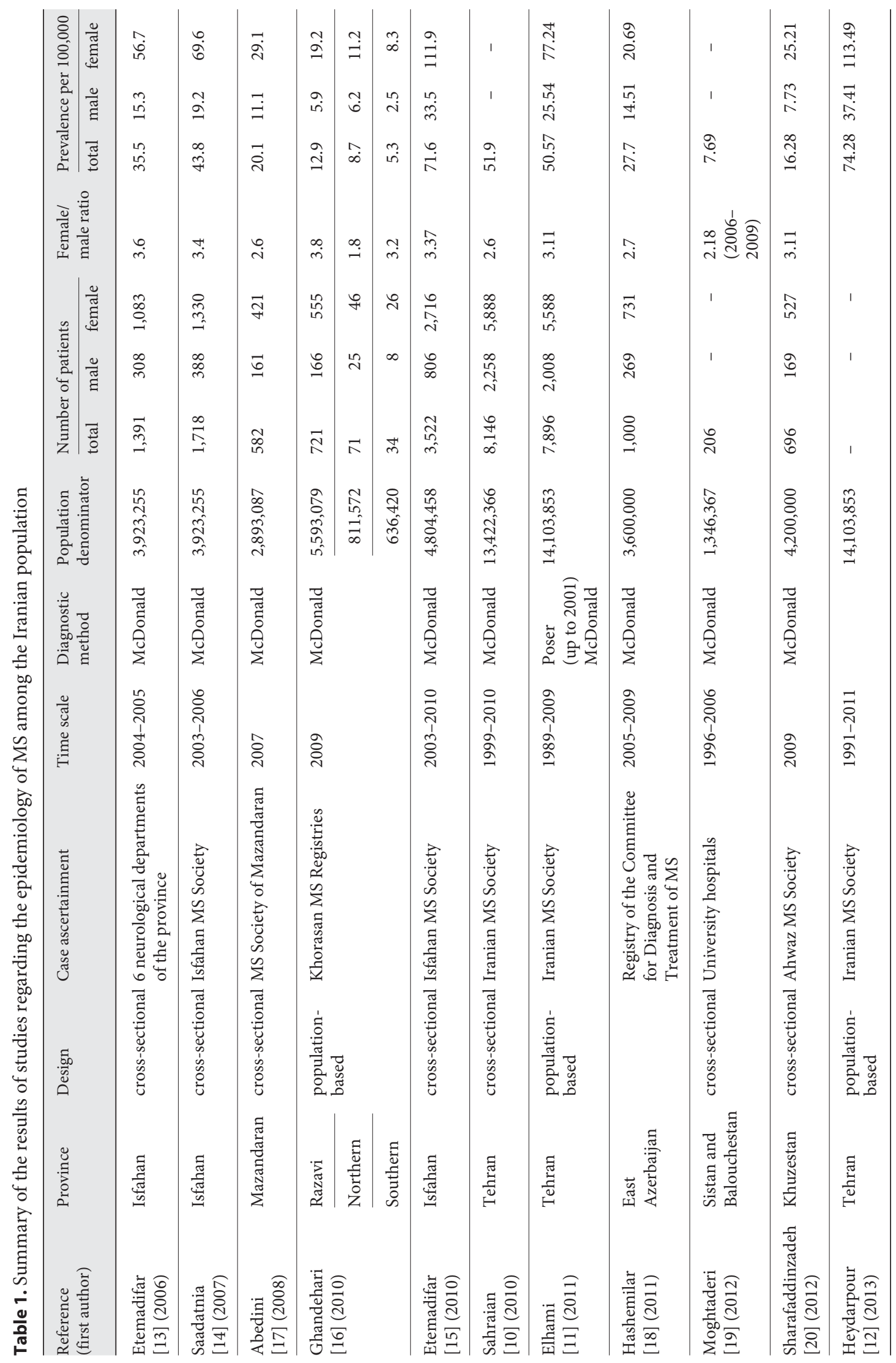


ages $20-39$ years. The main presenting symptom was visual impairment. RRMS $84.9 \%$ was much greater than other types $15.1 \%$. Of 8,146 patients, early-onset MS (age at onset $<16$ ) was $6.2 \%$, female/male ratio 3.3 , and lateonset disease (age $>50$ ) $0.5 \%$, female/male ratio 0.83 . Family history was positive $9.5 \%, 4.1 \%$ first-degree kinship.

Elhami et al. [11] in 2011 used 20 years of data in a well-defined and stable population, with age- and sex-adjusted incidence rates. The crude incidence rate increased from 0.68 in 1989 to 5.68 in 2005. The adjusted incidence rate increased from 0.68 in 1989 to 2.93 in 2008 . The ageadjusted prevalence rate was 50.57 , female/male ratio 3.11. Prevalence was highest in the age group 35-39 years in females and $40-44$ years in males. The mean age at onset of the disease was $27.53 \pm 8.25$ years and at diagnosis $29.02 \pm 8.60$.

More recently, Heydarpour et al. [12] in 2013 employed Joinpoint regression analysis to evaluate the trend of MS incidence, male/female ratio, mean age at disease onset, and the incidence of pediatric MS during two decades. The age-adjusted prevalence rates increased to 74.28 (113.49 in women and 37.41 in men). The age-adjusted incidence rate increased from 1.3/100,000 in 1991 to 6.6 in 2005. Overall, two incidence peaks were reported in this study; the annual percent increase for the first Joinpoint in 2004 was $12.8 \%$ in females and $12.5 \%$ for males. Sex ratio trend analysis among patients born between 1950 and 1995 has shown a significantly decreasing male/ female ratio. Pediatric MS (age at disease onset $<18$ ) was $7.23 \%$. The crude incidence in girls increased from 3.4 per million in 1991 to 33.2 in 2007. The second Joinpoint in 2008 was $12.3 \%$ in girls and $14 \%$ in boys.

\section{Isfahan}

Isfahan, in central Iran, is one of the largest and most populous provinces. Three epidemiological assessments on MS in Isfahan are reported in the literature. The first was carried out by Etemadifar et al. [13] for the year 2006, with an age-adjusted prevalence rate of 35.5 (54.5 in women, 14.9 in men) and a female/male ratio of 3.6. Ages 30-39 years had highest prevalence rates. The most common initial presentations were sensory and visual disturbances with 51.1 and $47.0 \%$ prevalence, respectively. $76.6 \%$ of patients were born in Isfahan province. A positive MS family history was $11.6 \%$.

Saadatnia et al. [14] in the second study in 2007 reported an incidence of 3.64 in 2005, an overall prevalence of 43.8 (69.6 among women and 19.2 among men), a female/male ratio of 3.4 , mean age at onset of $25.4 \pm 8.6$ years, RRMS 87.8\%, SPMS 6.4\%, and PPMS 5.7\%. Initial presentation was sensory and visual disturbances in 51.7 and $47.5 \%$ patients, respectively. Early- and late-onset MS was 5 and $1.1 \%$ of patients, respectively, and MS family history $12.2 \%$.

Etemadifar and Maghzi [15] in 2010 presented the most recent data for Isfahan: incidence 9.1 in 2009, standardized prevalence 71.6 , and female/male ratio 3.37 .

\section{Northeast Iran}

Khorasan is an area in northeast Iran, the largest province of the country, containing three subareas. Ghandehari et al. [16] in 2010 characterized three main trends for Razavi, northern and southern Khorasan provinces: total prevalence was 12.9, 8.7 and 5.3, respectively, and female/ male ratio $3.8,1.8$ and 3.2 , respectively.

\section{Mazandaran}

Mazandaran, one of the most densely populated cities of Iran, is in north Iran, south of the Caspian Sea. Data is only available in local scientific literature. In 2006 [17] a survey found a prevalence rate of 20.1, a female/male ratio of 2.6, and more common in third decade of life. The mean age at onset was $26.9 \pm 8.3$. Optical and sensory symptoms were more common at onset, with RRMS 71\%, SPMS 15.8\%, and PPMS 13.2\%.

\section{East Azerbaijan}

East Azerbaijan is a province in northwest Iran, bordering on the republics of Azerbaijan, Armenia and Nakhchivan. Iranians with Turkish ethnicity reside in Azerbaijan. We found only one study in 2011 by Hashemilar et al. [18] where the crude prevalence rate was 27.7 , female/male ratio 2.7, mean age of patients $33.4 \pm$ 8.9, RRMS 67.7\%, PPMS 2.8\%, and SPMS 11.2\%.

\section{Sistan and Balouchestan}

Sistan and Balouchestan, with Balouch ethnic background, is a large province in southeast Iran, bordering on Afghanistan and Pakistan. Moghtaderi et al. [19] in 2011 found an age-adjusted prevalence of 13.96 and an incidence of 2.67, female/male ratio 2.18, RRMS 65.8\%, SPMS $20 \%$, PPMS $6.7 \%$, and for progressive relapsing types $2 \%$, while $5.4 \%$ of patients had Devic's disease. MS was most common between 16 and 35 years of age. The EDSS score were $3.62 \pm 2.3$ for men and $2.70 \pm 2.1$ for women. Symptoms: numbness and other sensory disturbances (not visual), $39.8 \%$, motor dysfunction $28.6 \%$, optic neuritis $28.2 \%$, with positive family history in their first-degree relatives $6.2 \%$. 


\section{Khuzestan}

Khuzestan is in southwestern Iran, bordering on Iraq and the Persian Gulf, with both Persian and Arabic ethnic backgrounds. In 2012, Sharafaddinzadeh et al. [20] found total prevalence and incidence rates of 16.28 and 2.20 , respectively. The mean age was $31.4 \pm 8.50$ years and the mean age at disease onset was $26.85 \pm 7.63$ years, female/male ratio 3.11 and the mean EDSS score $2.89 \pm$ 1.91, with a higher score among the Arab population. RRMS 81.3\%, SPMS 12.2\%, PPMS 3.2\%. The most common presenting symptom was sensory signs and optic nerve involvement (78.2\%) followed by cerebellar symptoms (11.3\%) and motor deficit (10.3\%). $24.7 \%$ of the Arabian patients versus $15.9 \%$ of Persians experienced progressive patterns.

\section{Discussion}

\section{Epidemiological Reports and Their Limitations}

MS prevalence in Iran varies geographically, from 5.3 to $74.28 / 100,000$. The origin of such diversity is still elusive. Although different methodology, underestimation of the real incidence, and referral bias may be factors, there are different ethnicities - Kurds, Turks, Arabs and Balochs, - as well as different cultures and climates. This means great variations in diet and environmental exposures to many factors, both ancient traditional and novel modern.

Only 5 studies reported an age-adjusted prevalence [11-13, 15, 19], thus, age- and sex-adjusted prevalence and incidence should be considered in future reports. Moreover, there is a lack of complete data on incidence, clinical pattern, early-onset MS, late-onset MS, family history and prevalence of neuromyelitis optica among majority of reports. The minimum MS prevalence pertained to three eastern provinces of Khorasan [16] with the prevalence of 5.3-12.9, which is the lowest rate compared to the rest of the reports. This might be due to sampling flaw in this study. They only included patients who need interferon medication, so those who receive other immunosuppressive therapy or no therapy at all could have been overlooked [15]. However, overall, there has been a substantial rise in MS prevalence in a few decades. Possible explanations for such an increase are as follows. (1) The diagnosis of MS has improved due to more availability of MRI and paraclinical tests revising of the Mcdonald criteria, increasing numbers of neurologists and accidental diagnosis by physicians $[14,15]$. (2) The lack of early childhood exposure to common viral agents like
Epstein-Barr virus due to hygiene promotion after the 1981 Iran revolution, in line with the hygiene hypothesis [21].

The 20-year incidence trend of MS in Tehran, Iran, showed a huge increase over the past 18 years, from 0.68 in 1989 to $5.68 / 100,000$ in 2006 , an 8.3 -fold rise with generally higher prevalence rates among women [11].

A similar trend between 2007 and 2010 is reported from Isfahan province at the center of Iran, by which a dramatic increase in the prevalence and incidence of MS has been observed. Isfahan is a well-studied area, now globally known for its high and increasing incidence and prevalence of MS during the last decade, probably caused in part by changes in lifestyle and vitamin $\mathrm{D}$ deficiency $[15,22]$. Further features found among Isfahan MS patients include: (1) increasing female preponderance over the recent decade [23]; (2) highest rates among the 30- to 39-year age group, decreasing with increasing age; (3) high percentage in early-onset MS, and (4) the lower frequency of oligoclonal IgG bands in CSF of Isfahan MS patients than in the West [14]. Similar reports from Japan and Jordan may be evidence that oligoclonal IgG bands are less frequent in the CSF of Asian MS patients than MS patients in the West; however, more accurate studies are required to support this hypothesis [14, 24, 25].

Sistan and Baloochestan, a southeastern province, reported a relatively low prevalence of 13.96 [19]. Although their results are inconsistent with reports from Isfahan and Tehran, it is not surprising because the people who live in this area have different ethnicity and lifestyle compared to people in Isfahan and Tehran. The majority of people in Sistan and Baloochestan are from the Baloch ethnic group, which closely resembles the population residing in Pakistan, where MS is reported to be rare [2628]. Furthermore, nearly half of the population still lives in rural areas, while only 17 and $9 \%$ of the population of Isfahan and Tehran, respectively, live in rural areas. This factor alone plays an important role to show the major lifestyle differences between these regions. As mentioned, hypovitaminosis D has been shown to be associated with urban life.

The prevalence of 27.7 in east Azerbaijan, the northwest province was higher than its neighbor with the same population, Armenia, where MS has been reported to be $11.8 / 100,000$ individuals [29]. These results show that the prevalence of MS in Iran is more than its neighbors and indicates the role of genetics and environment in susceptibility to MS among the Iranian population.

Khuzestan, a southwest province, reported a prevalence of 18.50 among Persians versus 10.58 among Arabs. 


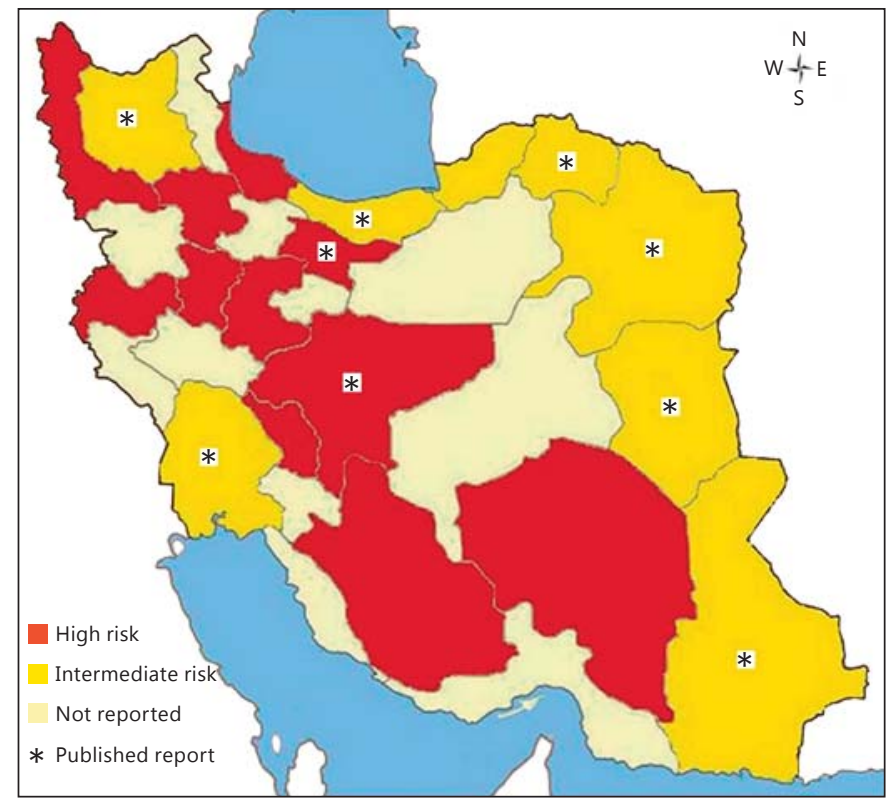

Fig. 1. Distribution of MS in Iran according to geographical classification. High risk: >30/100,000; intermediate risk: 5-25/100,000; low risk $<5 / 100,000$.

They attributed the origin of such differences to ethnic distinction of the aforementioned two groups residing in the same region. However, according to an anthropological study [30] there is no close genetic relationship between Iranian Arabs and Middle-Eastern Arabs, as Iranians who talk in Arabic have a genetic affinity to the rest of the Iranian ethnic groups. As we recently postulated [31], although the authors have shown that motor deficits and cerebellar findings are more frequent among Arabs than Persians, the overall patterns of MS presentation in both populations are the same with the predominance of optic nerve involvement, followed by sensory problems (28.6\%) and motor deficits - a pattern that resembles other reports from the rest of Iranian provinces. Engagingly, such a pattern is in obvious contrast with the clinical presentation of MS in other Middle-Eastern Arab countries neighboring Khuzestan, e.g. Iraq and Saudi Arabia, where motor deficits were the most common mode of presentation.

\section{Prevalence of MS across Iran and Geographical Distribution Hypothesis}

According to the north-south latitude hypothesis, Iran should be a low-risk area with a less than 5/100,000 MS prevalence. Of note, to date, the published epidemiology data of MS are insufficient for determining the geograph-
Table 2. Estimations of Iranian local MS registries about the number of their definitely diagnosed subjects

\begin{tabular}{lrrrrl}
\hline Province & $\begin{array}{l}\text { Population } \\
\text { denominator }\end{array}$ & $\begin{array}{l}\text { Total } \\
\text { patients }\end{array}$ & $\begin{array}{l}\text { Female } \\
\text { patients }\end{array}$ & $\begin{array}{l}\text { Male } \\
\text { patients }\end{array}$ & $\begin{array}{l}\text { Prevalence } \\
\text { per 100,000 }\end{array}$ \\
\hline West & & & & & \\
Azerbaijan & $3,080,576$ & 1,401 & 1,008 & 393 & 45.47 \\
Isfahan & $4,879,312$ & 4,541 & 3,527 & 1,014 & 93.06 \\
Kerman & $2,938,988$ & 1,029 & 811 & 218 & 35.01 \\
Kermanshah & $1,945,227$ & 850 & - & - & 43.69 \\
Charmahal & 895,263 & 830 & 780 & 50 & 92.71 \\
Hamadan & $1,758,268$ & 1,100 & 850 & 250 & 62.56 \\
Markazi & $1,413,959$ & 1,043 & 806 & 237 & 73.76 \\
Zanjan & $1,015,734$ & 338 & 220 & 118 & 33.27 \\
Fars & $4,596,685$ & 3,573 & 2,843 & 730 & 77.72 \\
Gilan & $2,480,874$ & 890 & 630 & 260 & 35.87 \\
Golestan & $1,777,014$ & 320 & 250 & 70 & 18.00 \\
& & & & &
\end{tabular}

Data gathered by Isfahan MS Society using a national query from the rest of Iranian MS societies and registries.

ical distribution of MS in Iran. Nevertheless, in order to show the overall pattern of MS in Iran, we also investigated unpublished data on the prevalence of MS from local MS registries across the country and presented them in line with previously reported data. Figure 1 indicates that Iran is an intermediate- to high-risk region and the bulk of studies in different provinces have rejected the geographical distribution hypothesis $[2,32]$.

\section{Role of Vitamin D Deficiency in Epidemiology of MS in Iran}

Available literature now supports the crucial role of vitamin D deficiency in MS. The high prevalence of vitamin D deficiency among women, youngsters and high school students, especially girls, has been found by recent studies in Isfahan and Tehran [33-35]. Although it has been found that veiled women have a low vitamin $\mathrm{D}$ status [36], a major changing lifestyle trend during the past decades, there is spread of urbanization, indoor living, air pollution, changes in diet, widespread use of sun screens, avoiding sun exposure for fear of skin cancer and concern about skin beauty, all of which are thought to cause vitamin $\mathrm{D}$ deficiency, with the latter two being more prevalent among females. Moreover, Iranian women spend less time in the sun, compared to men, which could also lead to vitamin $\mathrm{D}$ deficiency, but is independent of wearing a 
hijab or veil and therefore is mostly due to culture and lifestyle. These factors might have caused the gender difference in vitamin D deficiency in Iran $[7,37]$.

\section{Conclusion}

In conclusion, the incidence and prevalence of MS in Iran has been increasing rapidly, steadily, hugely, and mysteriously, especially in females. The overall epidemiologic picture is still unclear in a number of provinces, although at present unpublished estimations made by local MS registries are available (table 2). Future research should focus on determining the epidemiological features of MS in the overlooked provinces with different ethnicities, especially Yazd, Kurdistan, West Azerbaijan, Orumieh, and the northern areas of the country. Such an effort along with further research towards improvement of data on previously studied areas can enable a field to be opened up to identify the patterns of MS in varied genetic backgrounds and environments of Iran.

\section{Acknowledgment}

The authors are very grateful to Mr. Richard T. Murray (Medical Researcher) for revising and commenting on the manuscript.

\section{References}

$>1$ Frohman EM, Racke MK, Raine CS: Multiple sclerosis - the plaque and its pathogenesis. $\mathrm{N}$ Engl J Med 2006;354:942-955.

$>2$ Kurtzke JF: Epidemiologic contributions to multiple sclerosis: an overview. Neurology 1980;30:61-79.

3 Kurtzke JF: A reassessment of the distribution of multiple sclerosis. Acta Neurol Scand 1975; 51:137-157.

-4 Benamer HT, Ahmed ES, Al-Din AS, Grosset DG: Frequency and clinical patterns of multiple sclerosis in Arab countries: a systematic review. J Neurol Sci 2009;278:1-4.

$>5$ Pakpoor J, Ramagopalan S: Multiple sclerosis and the Iranian revolution: reply to the comment by Magzhi et al. Neuroepidemiology 2012;40:70.

6 Etemadifar M, Abtahi SH: Multiple sclerosis in Isfahan, Iran: past, present and future. Int J Prev Med 2012;3:301-302.

$>7$ Maghzi AH, Sahraian MA, Shaygannejad V, Minagar A: Is the Iranian revolution to blame for the increasing incidence of multiple sclerosis in Iran? Neuroepidemiology 2012;40: 68-69.

$>8$ Poser CM, Paty DW, Scheinberg L, McDonald WI, Davis FA, Ebers GC, Johnson KP, Sibley WA, Silberberg DH, Tourtellotte WW: New diagnostic criteria for multiple sclerosis: guidelines for research protocols. Ann Neurol 1983;13:227-231.

-9 McDonald WI, Compston A, Edan G, Goodkin D, Hartung HP, Lublin FD, McFarland $\mathrm{HF}$, Paty DW, Polman $\mathrm{CH}$, Reingold SC, Sandberg-Wollheim M, Sibley W, Thompson A, van den Noort S, Weinshenker BY, Wolinsky JS: Recommended diagnostic criteria for multiple sclerosis: guidelines from the international panel on the diagnosis of multiple sclerosis. Ann Neurol 2001;50:121-127.
10 Sahraian MA, Khorramnia S, Ebrahim MM, Moinfar Z, Lotfi J, Pakdaman H: Multiple sclerosis in Iran: a demographic study of 8,000 patients and changes over time. Eur Neurol 2010;64:331-336

11 Elhami SR, Mohammad K, Sahraian MA, Eftekhar H: A 20-year incidence trend (19892008) and point prevalence (March 20, 2009) of multiple sclerosis in Tehran, Iran: a population-based study. Neuroepidemiology 2011; 36:141-147.

12 Heydarpour P, Mohammad K, Yekaninejad MS, Elhami SR, Khoshkish S, Sahraian MA: Multiple sclerosis in Tehran, Iran: a Joinpoint trend analysis. Mult Scler (E-pub ahead of print).

13 Etemadifar M, Janghorbani M, Shaygannejad V, Ashtari F: Prevalence of multiple sclerosis in Isfahan, Iran. Neuroepidemiology 2006;27: 39-44.

14 Saadatnia M, Etemadifar M, Maghzi AH: Multiple sclerosis in Isfahan, Iran. Int Rev Neurobiol 2007;79:357-375.

15 Etemadifar M, Maghzi AH: Sharp increase in the incidence and prevalence of multiple sclerosis in Isfahan, Iran. Mult Scler 2011;17 1022-1027.

16 Ghandehari K, Riasi HR, Nourian A, Boroumand AR: Prevalence of multiple sclerosis in north east of Iran. Mult Scler 2010;16:15251526 .

17 Abedini M, Habibi-Saravi R, Zarvani A, Farahmand M: Epidemiology of multiple sclerosis in Mazandran province in 2007 (in Persian). J Mazandaran Univ Med Sci 2008;18: 82-87.

18 Hashemilar M, Savadi Ouskui D, Farhoudi M, Ayromlou H, Asadollahi A: Multiple sclerosis in east Azerbaijan, north west Iran. Neurology Asia 2011;16:127-131.
19 Moghtaderi A, Rakhshanizadeh F, ShahrakiIbrahimi S: Incidence and prevalence of multiple sclerosis in southeastern Iran. Clin Neurol Neurosurg 2013;115:304-308.

20 Sharafaddinzadeh N, Moghtaderi A, Majdinasab N, Dahmardeh M, Kashipazha D, Shalbafan B: The influence of ethnicity on the characteristics of multiple sclerosis: a local population study between Persians and Arabs. Clin Neurol Neurosurg 2013;115:12711275.

21 Maghzi AH, Marta M, Bosca I, Etemadifar M, Dobson R, Maggiore C, Giovannoni G, Meier UC: Viral pathophysiology of multiple sclerosis: a role for Epstein-Barr virus infection? Pathophysiology 2011;18:13-20.

-22 Alonso A, Cook SD, Maghzi AH, Divani AA: A case-control study of risk factors for multiple sclerosis in Iran. Mult Scler 2011;17:550555.

23 Maghzi AH, Ghazavi H, Ahsan M, Etemadifar M, Mousavi S, Khorvash F, Minagar A: Increasing female preponderance of multiple sclerosis in Isfahan, Iran: a population-based study. Mult Scler 2010;16:359-361.

24 El-Salem K, Al-Shimmery E, Horany K, AlRefai A, Al-Hayk K, Khader Y: Multiple sclerosis in Jordan: a clinical and epidemiological study. J Neurol 2006;253:1210-1216.

25 Tanaka K, Kujuro Y, Suzuki S, Tanahashi N, Hamada J, Nogawa S, Suzuki N: Clinical and laboratory features of in-patients with multiple sclerosis in a University Hospital in Tokyo from 1988 to 2002. Intern Med 2005;44:560_ 566.

26 Farjadian S, Naruse T, Kawata H, Ghaderi A, Bahram S, Inoko $\mathrm{H}$ : Molecular analysis of HLA allele frequencies and haplotypes in Baloch of Iran compared with related populations of Pakistan. Tissue Antigens 2004;64: 581-587. 
27 Wasay M, Ali S, Khatri IA, Hassan A, Asif M, Zakiullah N, Ahmed A, Malik A, Khealani B, Haq A, Fredrikson S: Multiple sclerosis in Pakistan. Mult Scler 2007;13:668-669.

28 Maghzi AH, Sahraian MA, Maghzi H, Shaygannejad V: Multiple sclerosis in Sistan and Baloochestan, south east Iran. Clin Neurol Neurosurg 2013;115:847-848.

29 Hakobyan ME, Angelova IB, Manvelyan HML: Comprehensive epidemiology of multiple sclerosis in Europe and Armenia: hidden pandemics? New Armenian Med J 2008;2:613.

30 Farjadian S, Ghaderi A: HLA class II genetic diversity in Arabs and Jews of Iran. Iran J Immunol 2007;4:85-93.
31 Fereidan-Esfahani M, Abtahi SH, Nourian SM, Etemadifar M: Multiple sclerosis among Iranian-Arabs and Persians of Khuzestan: is there any ethnic difference? Clin Neurol Neurosurg 2013 (E-pub ahead of print).

32 Kurtzke JF: A reassessment of the distribution of multiple sclerosis. Part 1. Acta Neurol Scand 1975;51:110-136.

33 Hovsepian S, Amini M, Aminorroaya A, Amini P, Iraj B: Prevalence of vitamin D deficiency among adult population of Isfahan City, Iran. J Health Popul Nutr 2011;29:149155.

34 Moussavi M, Heidarpour R, Aminorroaya A, Pournaghshband Z, Amini M: Prevalence of vitamin D deficiency in Isfahani high school students in 2004. Horm Res 2005;64:144-148.
5 Rabbani A, Alavian SM, Motlagh ME, Ashtiani MT, Ardalan G, Salavati A, Rabbani B, Shams S, Parvaneh N: Vitamin D insufficiency among children and adolescents living in Tehran, Iran. J Trop Pediatr 2009;55:189191.

36 Guzel R, Kozanoglu E, Guler-Uysal F, Soyupak S, Sarpel T: Vitamin D status and bone mineral density of veiled and unveiled Turkish women. J Womens Health Gend Based Med 2001;10:765-770.

37 Pakpoor J, Ramagopalan SV: Multiple sclerosis and the Iranian revolution. Neuroepidemiology 2012;38:122. 